\title{
Study of radio energy transmission system based on magnetic coupling resonance
}

\author{
Xiangfei Meng ${ }^{\mathrm{a}}$, Chenxi Zhu ${ }^{\mathrm{b}}, \mathrm{Xin} \mathrm{Li}^{\mathrm{c}}$, Huigang $\mathrm{Xu}^{\mathrm{d}}$ \\ Changshu Institute of Technology, School of Electrical and Automation Engineering, Changshu \\ 215500, China \\ a'Email: mxf0316@163.com, ${ }^{\mathrm{b} E m a i l: ~ z c x @ c s l g . c n, ~}{ }^{\mathrm{c} E m a i l: ~ I x @ c s l g . c n, ~}{ }^{\mathrm{d} E m a i l: x h g @ c s l g . c n}$
}

\begin{abstract}
Keywords: Magnetic coupling resonance, wireless transmission, transmitting module, receiving module
\end{abstract}

Abstract. As a new power supply mode, wireless energy transmission has a very broad application prospect, which has been widely concerned by academia and industry. In this paper, based on the principle of magnetic coupling resonance, analyzed the technology and designed a set of wireless energy transmission system and summarized the function and principle of each module. The wireless transmission device is made according to the principle.

\section{Introduction}

In recent years, with the development of small mobile devices and the traditional cable energy supply problems, the traditional power supply mode has been unable to meet people's requirements for mobility and special occasions, wireless power transmission technology has become a research hotspot. The application of wireless transmission technology will be very extensive, in the field of biomedical equipment can be non-contact power supply; underwater, under the mine equipment for wireless power supply. wireless power supply for portable equipment, can make people's life more convenient, so it is necessary to design efficient and convenient wireless power supply equipment. At present, most of the wireless transmission mode is based on electromagnetic induction coupling technology, the technology is simple, but the disadvantage is that the transmission distance must be short, and the efficiency is low. And the application of magnetic coupling resonance technology can greatly improve the electromagnetic induction coupling technology, that is to achieve a medium range of energy efficient transmission.

\section{The principle of magnetic coupling resonance technology}

Magnetic resonance wireless transmission technology is the use of two oscillators with the same resonant frequency, in a certain distance, the magnetic field coupling to produce a resonance, to achieve power transmission, energy transfer medium is the medium high frequency magnetic field. The basic idea is that two coils with the same resonant frequency can be realized by coupling resonance and the energy exchange with high efficiency, and the interaction between different frequency coils is weak.

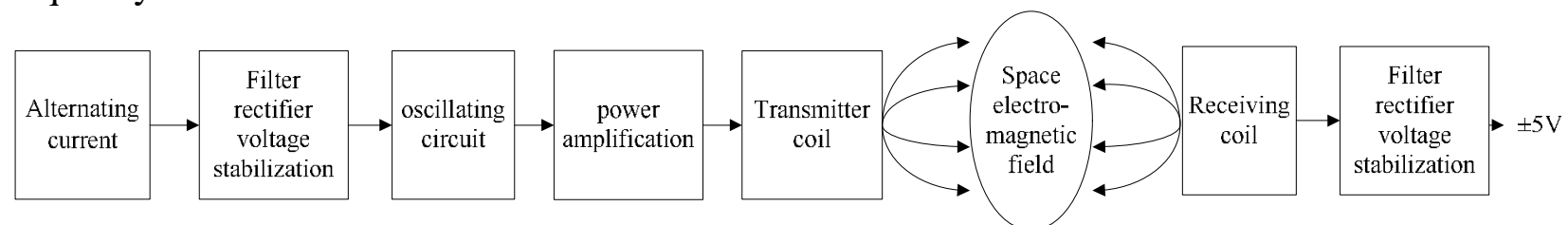

Fig.1 The schematic diagram of magnetic coupling resonance system

As shown in Fig.1. System is mainly composed of two same resonance frequency oscillator, oscillating circuit, power amplification circuit, rectifier, regulator and filter circuit, through the magnetic coupling resonance, the energy from the transmitter coil through the "tunnel of space" is passed to the receiving coil, then filtering, rectifier, regulator of $\pm 5 \mathrm{~V}$ power supply required to obtain a test circuit. 


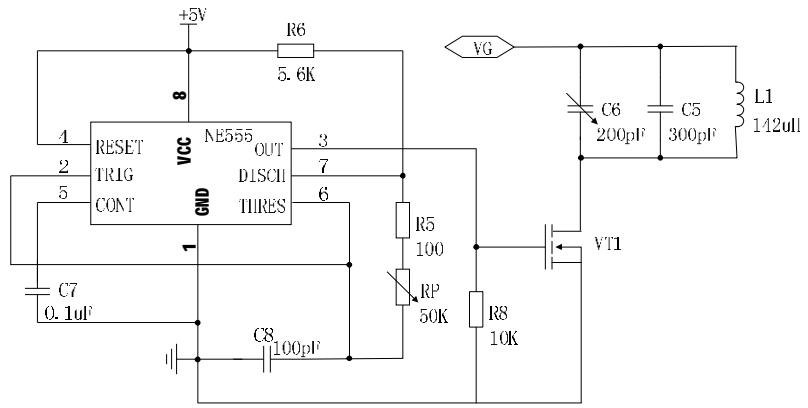

Fig. 2 The transmitting circuit

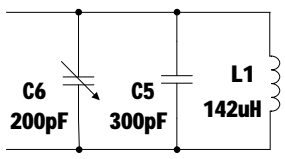

Fig.3 The resonant circuit

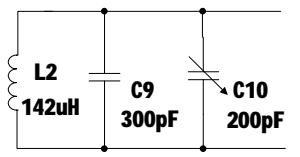
uit

Transmission circuit consist of oscillation signal generator and power amplifier circuit, as shown in Fig.2. Applied by NE555 oscillation frequency is about $510 \mathrm{Khz}$ signal generator, providing incentives for power amplifier circuit signal; Resonance power amplifier composed of LC resonance circuit and switch tube IRF840. Oscillation coil with a diameter of $0.80 \mathrm{~mm}$ according to the requirement of the enameled wire tightly wound 20 about $6.5 \mathrm{~cm}$ in diameter, the measured inductance value is about $142 \mathrm{uH}$, by the known, when resonance at $510 \mathrm{KHZ}$, instead of parallel capacitor C5, C6, approximately $680 \mathrm{pF}, 470 \mathrm{pF}$ fixed capacitors in parallel are available a $200 \mathrm{pF}$ adjustable capacitance, easy to adjust the resonance frequency.

When power amplifier circuit of frequency selective resonant frequency and the excitation signal frequency at the same time, the power amplifier in resonance, the maximum voltage and current in coil, resulting in a maximum of alternating electromagnetic field. When receiving coil and the transmitter coil near the output voltage is induced in the receiving coil, when the resonance frequency of the receiver coil circuit and transmitting frequency resonate at the same time, the voltage of the maximum value. Constitute the resonant circuit is shown in Fig.3. In fact, the transmitter coil circuit and receiving coil circuit are in a state of resonance, havig the best effect of energy transmission.

\section{Launch circuit design}

The transmitter circuit of the wireless charging device is shown in Fig.4, which is mainly composed of a limiting circuit, a dead time forming circuit, a gate isolating circuit, an inverter circuit, a transmitting coil, etc.. In the circuit on the electric moment due to disturbance signal is detected after the transformer T3, increased by T3 and sent to the input end of the voltage limiter circuit, signal voltage is limited below $5 \mathrm{~V}$ and sent to the comparator 3 feet by 7 feet, TL3116, 8 complementary output square wave signal, is sent to a $74 \mathrm{HC} 132$ circuit to generate a complementary form of dead zone a certain delay wave signal, then the signal through the isolation driving circuit of power amplifying gate drive, half bridge circuit, the LC occurs in a certain frequency range, so the transmitter coil generates a magnetic field, because of mutual inductance, the electromagnetic field is received by the receiving coil, the resonant coupling of electromagnetic field transformation of $\mathrm{AC}$ current, and then sent to the high-frequency rectifying circuit of high frequency AC power to DC, the capacitor filter circuit to DC power becomes more smooth.

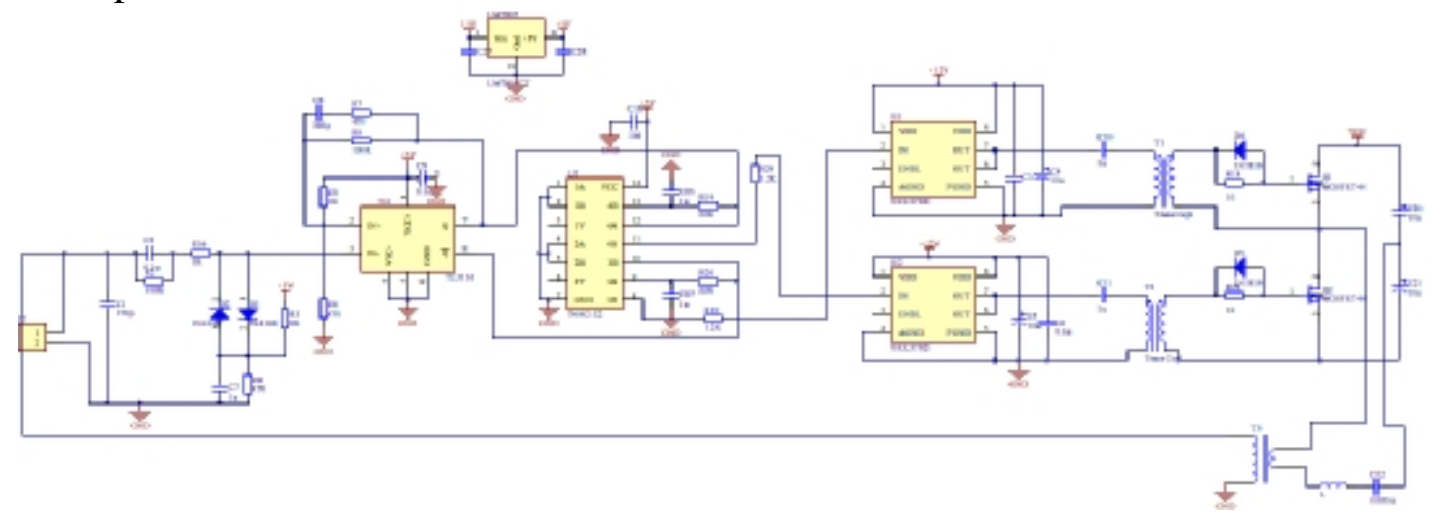

Fig.4 Transmitter end circuit diagram for wireless transmission device 


\section{Receiving circuit design}

In Fig. 5 for the receiving circuit, the receiving coil is received in the energy, and the first through the full bridge rectifier module and filter capacitor part of a more stable DC power. The DC energy signal can be changed with the change of the distance between the coil, the transmitting voltage and the working frequency of the system. Therefore, the DC/DC module is usually used to perform the energy conversion before receiving the load.

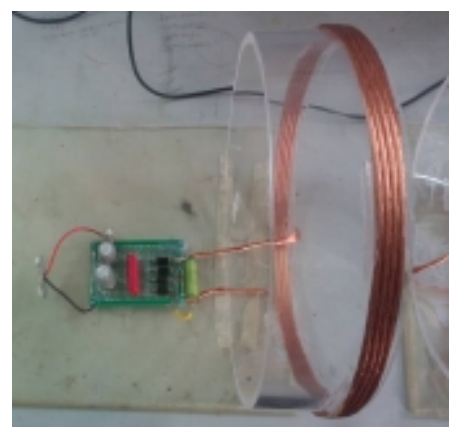

Fig.5 Receiving circuit physical map

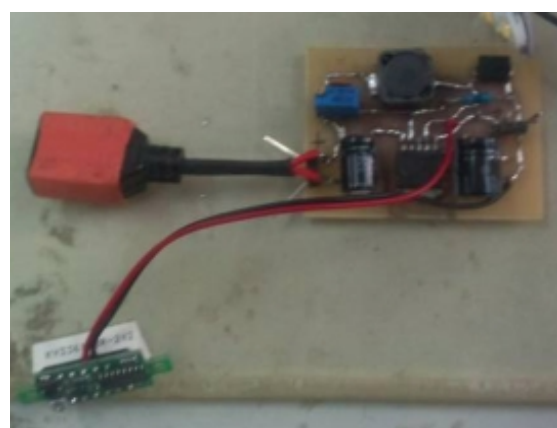

Fig.6 Rectifier control module and DC/DC module

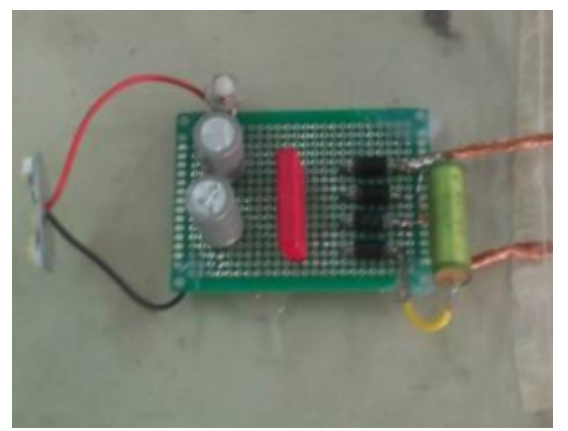

As shown in Fig.6, the rectifier control module and DC/DC module are used in this design. The module uses the LM2596 chip for the buck program. Its input range is $4.5-35 \mathrm{~V}$, the output range is $1.25-30 \mathrm{~V}$, the maximum output current can reach $3 \mathrm{~A}$, which can meet the charging and power supply requirement of common equipment. This design can use the DC/DC module output positive 5V DC power to the mobile phone socket to achieve wireless charging.

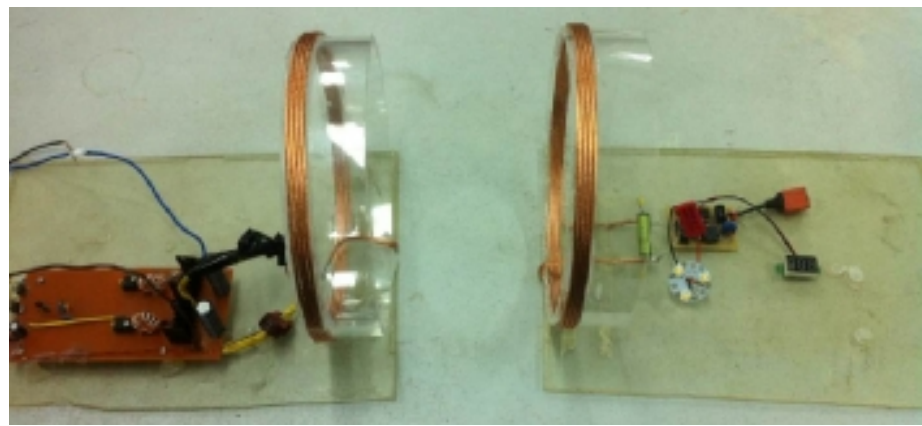

Fig.7 Physical model of magnetic resonance wireless transmission

\section{Experimental analysis of energy transfer efficiency}

Compared with the traditional cable transmission, the resonant wireless energy transmission system has its inherent complexity, and its transmission effect is very easy to be affected. The influence of the distance between the transmitter and the receiver is measured by experiment. The transmission voltage of this experiment is $5 \mathrm{~V}$, the transmission distance is increased, the receiving and transmitting power are calculated respectively. The measured experimental data are shown in Table 1. 
Table 1 the relationship between transmission distance and power

\begin{tabular}{|c|c|c|c|c|c|c|c|}
\hline $\begin{array}{c}\text { Distance } \\
\text { /CM }\end{array}$ & $\begin{array}{c}\text { Receiving } \\
\text { Voltage/V }\end{array}$ & $\begin{array}{c}\text { Receive } \\
\text { Current/A }\end{array}$ & $\begin{array}{c}\text { Received } \\
\text { Power/W }\end{array}$ & $\begin{array}{c}\text { Emission } \\
\text { Voltage/V }\end{array}$ & $\begin{array}{c}\text { Emission } \\
\text { Current/A }\end{array}$ & $\begin{array}{c}\text { Transmitting } \\
\text { Power/W }\end{array}$ & $\begin{array}{c}\text { Efficiency } \\
/ \%\end{array}$ \\
\hline 2 & 15.6 & 0.147 & 2.2932 & 5 & 0.58 & 2.9 & 79.1 \\
\hline 4 & 16.5 & 0.156 & 2.574 & 5 & 0.71 & 3.55 & 72.5 \\
\hline 6 & 16.85 & 0.164 & 2.7634 & 5 & 0.88 & 4.4 & 62.8 \\
\hline 8 & 17.01 & 0.166 & 2.82366 & 5 & 1.08 & 5.4 & 52.3 \\
\hline 10 & 16.39 & 0.16 & 2.6224 & 5 & 1.27 & 6.35 & 41.3 \\
\hline 12 & 15.18 & 0.148 & 2.24664 & 5 & 1.43 & 7.15 & 31.4 \\
\hline 14 & 13.61 & 0.133 & 1.81013 & 5 & 1.56 & 7.8 & 23.2 \\
\hline 16 & 11.7 & 0.114 & 1.3338 & 5 & 1.68 & 8.4 & 15.9 \\
\hline 18 & 9.47 & 0.092 & 0.87124 & 5 & 1.77 & 8.85 & 9.8 \\
\hline 20 & 8.13 & 0.079 & 0.64227 & 5 & 1.82 & 9.1 & 7.1 \\
\hline 30 & 4.21 & 0.041 & 0.17261 & 5 & 1.91 & 9.55 & 1.8 \\
\hline 40 & 2.224 & 0.021 & 0.046704 & 5 & 1.95 & 9.75 & 0.5 \\
\hline 50 & 1.298 & 0.012 & 0.015576 & 5 & 1.97 & 9.85 & 0.2 \\
\hline
\end{tabular}

From the experimental data coordinate diagram shown in Fig.8, it can be seen that in other conditions, the transmission efficiency of the system decreases with the increase of transmission distance, when the distance reaches about half meter, the efficiency is basically zero.

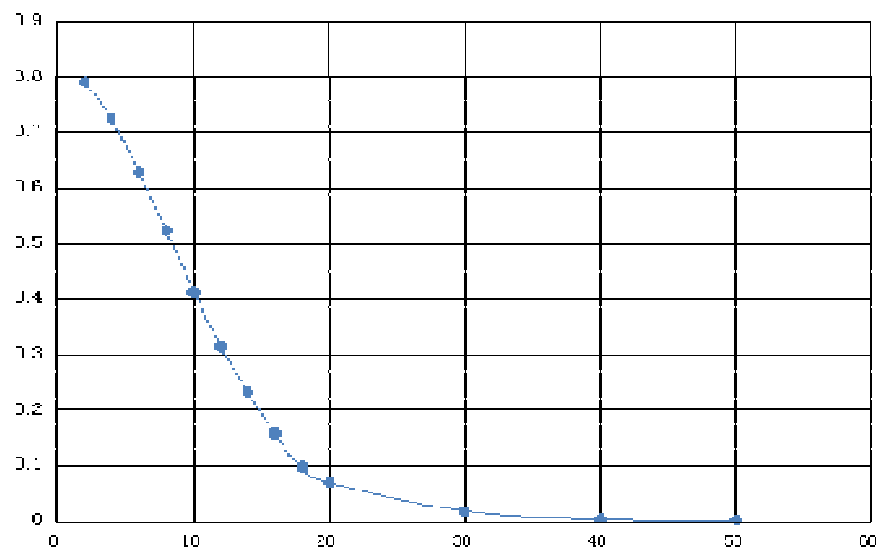

Fig.8 Relationship between efficiency and distance

\section{Conclusion}

Compared with the conventional contact transmission, wireless transmission is the biggest characteristic of transmission platform "whenever and wherever possible existence", in addition to the cable chains". In addition, the transmission efficiency of the wireless transmission system based on the magnetic coupling resonance is higher, the distance between the transmitting coil and the receiving coil is the distance between the coil and the coil. Using magnetic coupling resonance technology to design the actual power supply device and equipment, is conducive to promoting the popularization of wireless transmission technology as soon as possible.

\section{Acknowledgements}

This paper is supported by the Jiangsu Province research joint innovation fund project (NO. BY2014075) and by new introduction of teacher research and start fund project (NO. KYZ2014010Z) 


\section{References}

[1] He Ruihua. Discussion for development of low voltage apparatus in smart grid system[J]. Low voltage apparatus. 2011(1):1-5.

[2] Yang Qingxin, Chen Haiyan,et al. The research progress of contactless power transfer technique[J]. Journal of Electrotechnics. 2010(7):6-13.

[3] Karalis A, Joannopoulos J D, Soljacic M. Efficient wireless non-radiative mid-range energy transfer[J].Annals of Physics, 2008, 323: 34-48. 